\title{
Appendix D: Technical University of Turin $^{1}$
}

\section{Shiva Loccisano, Emilio Paolucci and Riccardo Ricci (Technical University of Turin, Italy)}

\section{D.1 INTRODUCTION OF THE UNIVERSITY AND ITS REGIONAL CONTEXT}

The Technical University of Turin (in Italian 'Politecnico di Torino'; PoliTo for short) is a part public engineering university based in Turin, Italy. Founded in 1859, it is Italy's oldest technical university, formerly being known as the 'School of Application for Engineers'. From when it was first established until approximately 1999, its main role was to transmit knowledge within the local ecosystem through highly educated and skilled students and graduates. Since 1999, after the founding of the university incubator, the role of the university has progressively changed. PoliTo nowadays is a more complex institution that focuses not only on teaching but also on scientific research, technology and knowledge transfer.

According to the world university rankings, in 2018, PoliTo is in 387th position in the QS World University Ranking and 33rd position in the 2018 QS World University Ranking for Engineering and Technology. In addition, the QS World Graduate Employability Ranking has placed PoliTo in first position, with reference to the 'Graduate Employment Rate' indicator (within 12 months of graduation), attesting to the quality of its education and its reputation among firms. Table D. 1 summarises some information related to PoliTo for the years $2011^{2}$ and 2017 .

1 The (re-)numbering of sections, tables and graphs in this appendix was done by this book's editors, as well as other minor edits to ensure text and format consistency across appendices $\mathrm{B}$ to $\mathrm{F}$.

2 We opted for 2011 as a complete set of data was available for that year. 
Table D.1

PoliTo key facts and figures (2011 and 2017)

\begin{tabular}{lrr}
\hline & 2011 & 2017 \\
\hline Total Budget & $€ 186.1 \mathrm{M}$ & $€ 250 \mathrm{M}^{1}$ \\
\# academic staff (FTE) & 887 & 878 \\
\# students & 28631 & $31000(15 \%$ foreign students $)$ \\
\# research publications $^{2}$ & $2290(2013)$ & 2810 \\
$\%$ of top 10\% publications & & $15 \%$ \\
\hline
\end{tabular}

Notes: $\quad{ }^{1}$ Approx. 50\% originates from the Italian Ministry, 10\% from students' tuition fees and $40 \%$ from competitive research grants. ${ }^{2}$ Sources: ETER, Elsevier (SCOPUS database and SciVal), PoliTo database.

Although PoliTo performs most of its activities in the Turin metropolitan area, which is identifiable at a NUTS3 level, it is also active at a regional level. In 2017, the Regional Innovation Scoreboard (RIS) classified the Piedmont regional economic system as 'moderate + innovator', a classification that is very close to the upcoming grade (Strong Innovation Leader). The Piedmont region has several local firms which have strong research and innovation capabilities. RIS, in fact, shows that Piedmont has a relative advantage in the 'private R\&D expenditure' ${ }^{3}$ and 'SMEs innovating-in-house' indicators, with respect to other European regions.

Until approximately 1990, Piedmont and the Turin metropolitan area were characterised by the presence of the Fiat ${ }^{4}$ car-maker and its suppliers, as well as other large companies, such as Telecom Italia, Leonardo, Thales, Comau, Magneti Marelli and Ferrero. A wide chain of small businesses clustered around these large companies that operate in industries such as automotive, aeronautics, telecommunications and textile. Over time, the region has developed high innovation capabilities in these technological specialisation areas and a strong network of relationships among local industrial actors. PoliTo has always had close relationships with such large firms, in the form of partnerships and research collaborations, as well as through the supply of a constant flow of skilled engineers and architects.

The engagement and impact of PoliTo within the regional ecosystem have progressively increased from the 1990s, when the Turin area underwent a profound transformation that was caused by the Fiat crisis and the contemporaneous prolonged crisis of the Italian economy, increasing international competition, together with the joint decline of traditional industries, and the rise of the knowledge-based economy. In those years, large companies started

Regional Innovation Scoreboard, 2017.

4 Now Fiat Chrysler Automobile (FCA). 
moving their production to other countries and, consequently, the SMEs and the entire Turin economy were affected to a great extent. In order to overcome the crisis, the regional government developed a new strategic plan, ${ }^{5}$ with the aim of defining priorities, in terms of regional specialisations. The plan of the regional government gave a key role to the university system in this technological specialisation process. In that period, and in line with the regional strategic plan, PoliTo adopted a number of actions that were aimed at supporting the ongoing transformation towards a more diversified economic system, adding more cross-disciplinary general-purpose technologies (namely ICT) to the traditional manufacturing competences, while favouring the creation of such new industries as biotechnology, mechatronics, biofuel, advanced materials etc. In so doing, PoliTo built formal and stable collaborations with local firms, in the form of collaborative research, contract research, consulting, joint ventures, joint participation in EU funded programmes, etc. PoliTo also founded two different research centres (ISMB ${ }^{6}$ in 2000 and SITI $^{7}$ in 2002) in partnership with several large companies (Telecom Italia, Motorola, Fiat, ST Microelectronics, etc.) and local public institutions (e.g. the Compagnia di San Paolo Foundation). The mission of these centres was to develop internet-based technologies and to promote their adoption in such traditional sectors and in areas such as logistics, territorial safety, environmental protection and urban renewal.

The following sections offer further detail about these changes and the contribution of PoliTo to its regional ecosystem has been divided into four impact categories as provided by the RIIA ${ }^{8}$ framework.

\section{D.2 REGIONAL ORIENTATION, STRATEGIC DEVELOPMENT, AND KNOWLEDGE INFRASTRUCTURE}

Over the last 20 years, the key objectives of the regional government have been to renew traditional manufacturing industries and to diversify through the introduction of other industries. The decision to work on how to diversify the regional economic system was also a response to the international economic crisis of 2007-2008 and to the increasing international competition. The regional government has always considered PoliTo and UniTo, ${ }^{9}$ thanks to their

\footnotetext{
DOCUP (Documento unico di Programmazione) in 2000-2006.

Istituto Superiore Mario Boella.

Istituto Superiore sui Sistemi Territoriali per l'Innovazione.

Research and Innovation Impact Assessment.

9 The other large university in the Turin area, which focuses on Basic Sciences and Humanities (www.unito.it).
} 
high-quality research and knowledge transfer, as a key means of developing new technological specialisation trajectories. PoliTo has collaborated with the region in the development of its strategic plans and has always been a key partner in their implementation.

As a result, PoliTo has continuously renewed its overall strategy over the last 20 years, adding new activities to the core missions of research (mostly in collaboration with local industrial players) and education for graduate students and industry members. Similarly, knowledge and technology transfer, once exclusively oriented towards large companies to help them solve specific technical problems, has changed to become more coherent with regional policies. The exploration and exploitation of new technological trajectories that were absent in the local industrial ecosystem has helped create new regional specialisation opportunities. For example, in 2005, a key initiative was undertaken, that is, the creation of a business research centre aimed at attracting the research laboratories of local and international firms (e.g. General Motors, Pirelli, Microsoft, Vishay, FEV) to the PoliTo campus in order to support the 'cross-fertilisation' of knowledge from different domains and to develop new forms of industry - university collaboration.

PoliTo also introduced innovations to the organisational model of the Technology Transfer Office (TRIN) by reinforcing its ability to manage administrative and legal issues, introducing a research centre on Entrepreneurship and Innovation, which provides research and methodological support to technology transfer activities, and supports the policy and adoption of new practices, and a new unit (called 'Lab TT') that acts as a liaison between TRIN and the traditional departments (Figure D.1).

The last step undertaken in order to develop a critical mass in new research domains was the creation, in 2016, of 11 cross-departmental research centres, which have been closely engaged with firms and other actors in the regional ecosystem and have focused on the development of breakthrough technologies (i.e. additive manufacturing, photonics, power conversion, autonomous vehicles, etc.). As a final result of all these transformations, PoliTo now has a dual strategy in place, whereby technology-push is mixed with market-pull, based on two main pillars: (1) generation and exploration of new knowledge for long-term innovation and diversification of the regional economic system; (2) exploitation of research results to drive the economic and societal impact in the medium to short term.

Overall, these actions have been consistent with PoliTo's strategy of leveraging knowledge accumulated from research over time at the local level through applied research projects developed in collaboration with firms. This approach has thus contributed to strengthening the network of relationships within the local ecosystem and to increasing the technological proximity between PoliTo and local firms. The ability of PoliTo to support the existing 


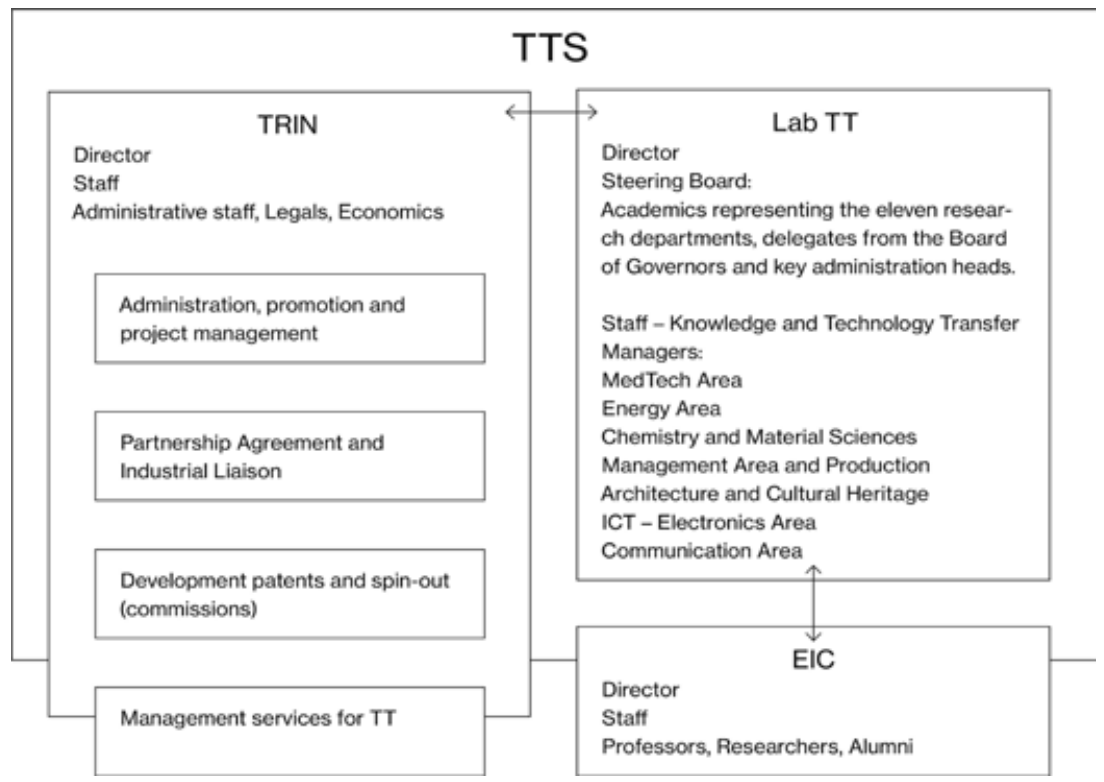

Figure D.1 TTO organisation model at PoliTo

technological specialisation has been confirmed by recent research ${ }^{10}$ that shows how, in the period from 1999 to 2013, PoliTo and firms in Piedmont patented in the same technological areas, and that PoliTo accompanied large companies in the exploitation of new technological areas. Since 2014, a new direction of strategic development has helped to strengthen and diversify the region's technological specialisation, as PoliTo has started to file patents in new technological areas. This strategic development has been consistent with regional policies. Indeed, the focus of both regional 'platforms' and 'clusters' programmes has also been targeted to new emerging fields (such as biotechnologies, health sciences and mechatronics) in order to generate a 'cross-fertilisation' between traditional and new industries. At the same time, PoliTo's exploration approach has been consistent with the European Commission's objective of stimulating 'excellence in research'. In fact, PoliTo has started exploring new technologies and has developed new competencies (i.e. nanomaterials, bioengineering, energy storage, etc.), mainly by conduct-

10 Colombelli, A., De Marco, A., Paolucci, E., Ricci, R. and Scellato G. (2019), University technology transfer and the evolution of regional specialisation: the case of Turin, PoliTo working paper. 
ing research projects financed within the EU's 6th, 7th and 8th Framework Programmes.

The PoliTo strategy has had a significant impact on the regional ecosystem. The number of patents and spin-offs is increasing and collaboration with companies is growing (there are 36 long-term partnership agreements in place, the industry co-patent rate is $53.7 \%$ and the co-publication rate with industry is $6.8 \%$ ). PoliTo, together with its I3P Incubator, has been a key leading actor in creating a new entrepreneurial ecosystem in the Turin area. ${ }^{11}$ In fact, on average, 54 from every 1000 graduates found a new company. PoliTo also plays a key role in connecting the regional innovation system with international research networks. For example, it has been engaged in 12 European Research Projects funded by the ERC, of which ten have been approved as 'Principal Investigator' and two as 'Partner Institutions'. It is also participating in two EU FET Flagship Initiatives: Graphene and the Human Brain Project. Moreover, $45.4 \%$ of the publications in scientific journals have had at least one foreign author. These data demonstrate the strategic intention of the institution to adopt an international orientation and to create links between regional and international actors.

\section{D.3 EDUCATION AND HUMAN RESOURCES DEVELOPMENT}

Currently, PoliTo provides teaching to approximately 33100 students, of which around $15 \%$ are foreign students ${ }^{12}$ and $45 \%$ are Italians from outside Piedmont (students tend to choose PoliTo because its employability rate is higher than several other Italian universities). Around two-thirds of the courses are given in English, and courses are provided to around $700 \mathrm{PhD}$ students (the funding of many $\mathrm{PhD}$ grants comes from local and international companies).

The quality of education has been rated highly by students. A recent graduate survey, conducted by AlmaLaurea ${ }^{13}$ in 2017, revealed that nine students out of ten appreciate their degree course (Figure D.2a). Similar results were obtained in terms of satisfaction with the degree for the job performed after graduation (Figure D.2b).

11 Colombelli, A., Paolucci, E. and Ughetto, E. (2017), Hierarchical and relational governance and the life cycle of entrepreneurial ecosystems. Small Business Economics, 1-17.

12 Selection procedures also take place in Buenos Aires, Santiago and Beijing.

13 AlmaLaurea is an association that was founded in 1994 which annually collects statistics from the associated universities. It is compulsory for almost graduated students to submit an online survey before they can graduate. This ensures a high number of responses. 
The 2017 QS World Graduate Employability Ranking, pertaining to the 'Graduate Employment rate' indicator assigned first position in the world to PoliTo, and the AlmaLaurea survey revealed that employment within a year after graduation was close to $90 \%$. PoliTo also promotes the retention of graduate students in the Turin area. In fact, the same AlmaLaurea survey revealed that $71.1 \%$ of graduate students ( 7 out of 10 ) would be willing to work in the Turin Area, and this choice was followed by the province of residence $(62.9 \%)$, North Italy $(62.2 \%)$, the Piedmont Region $(62.0 \%)$ and another European country $(60 \%)$. Moreover, the university invests in retaining promising researchers as it has decided to increase the standard national grant for $\mathrm{PhD}$ students by $25 \%$. PoliTo is also actively involved in connecting education with industry, since more than $90 \%$ of its students participate in a traineeship in a company.
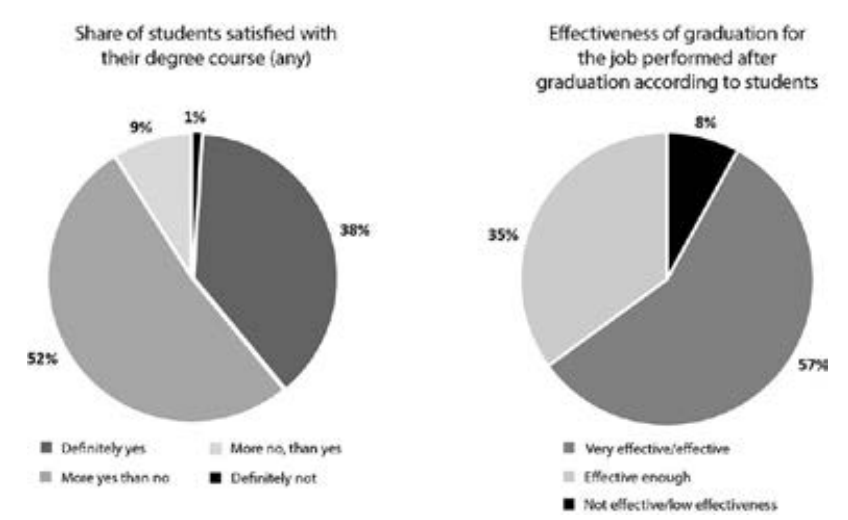

Source: $\quad$ AlmaLaurea 2018 Survey - 2017 Graduates’ Profile.

Figure D.2a and D.2b Education quality

In order to increase the quality of education, PoliTo has also developed its internationalisation activities, thanks to the 463 partnership collaborations with other universities, and more than 1000 incoming and 1000 outgoing Erasmus students.

However, the education carried out by PoliTo is not only addressed to students, but also to members of industry and graduate students. In particular, PoliTo collaborates with local industrial associations to provide post-degree courses in order to develop new competencies for managers and employees. Currently, it provides such teaching programmes, focused on new technologies, to about 550 students (including graduate students and managers). 
In recent years, PoliTo has strategically invested in entrepreneurship education to foster an entrepreneurship mind-set in students and young researchers. Around $4-5 \%$ of bachelor students attend at least one course on entrepreneurship or innovation. PoliTo also offers several elective courses on entrepreneurship and innovation at the BSc, MSc and $\mathrm{PhD}$ levels. For example, since 2014, it has hosted the European Innovation Academy, an intensive summer entrepreneurship school in which about 500 international students (including students from the Politecnico di Torino), guided by renowned mentors and with the sponsorship of multinational firms and international institutions, are challenged to transform their ideas into a technology start-up while working in a multidisciplinary and international team. Since 2015, in partnership with CERN and the Agnelli Foundation, it has offered Innovation for Change, an entrepreneurship programme for about 60 students ( $\mathrm{PhD}$ and MBA) with the objective of identifying solutions to long-term challenges proposed by large companies. Moreover, it has very recently created a 'fab lab'-like programme for bachelor students and has received funding from MIUR ${ }^{14}$ for new, cross-disciplinary educational programmes. A common feature of all these courses is the fact that students' activities start from challenges made available by companies, research centres, universities, start-ups, and so on.

\section{D.4 RESEARCH, TECHNOLOGICAL DEVELOPMENT, AND KNOWLEDGE TRANSFER}

PoliTo has an average of $14 \%$ of the total top annual publications cited in their own specific scientific field. This demonstrates the high-quality research of its faculty. Similarly, the institution is committed to transferring the knowledge obtained from research to the ecosystem. In fact, PoliTo's Statute clearly recognises the importance of the valorisation of research results. The university's 'third mission'; its contribution to economic and social development of the region, has been managed by the TRIN, supported by the I3P Incubator.

PoliTo's Proof of Concept (PoC) programme, a new key initiative, has determined a further positive impact that has accelerated the pace of spin-off creation and growth. PoliTo acts as a proactive investor by addressing the most critical phase in the innovation process between invention (when Intellectual Property is created) and technology development, when commercial concepts are created and verified, and proper markets are identified. The PoC pro-

14 MIUR is a funding instrument of the Italian Ministry of Education, Universities and Research to support excellence in Italian universities (https://www.miur.gov.it/ dipartimenti-di-eccellenza). 
gramme has two annual calls and funds of $€ 50000$. An analysis of completed PoCs reveals that the programme has increased the Technology Readiness Level by two stages on average, from around 3 to 5; it has also created new opportunities for patent licensing and start-up creation, proving that PoliTo improved its ability to transfer research into industrial applications. In fact, the spin-offs created in the 2017-2018 period received around $€ 2$ million (before incubation), with a post money value of around $€ 10$ million. It is also worth noting that the CEOs of such start-ups were all post-docs who had attended the new courses on entrepreneurship creation at PoliTo.

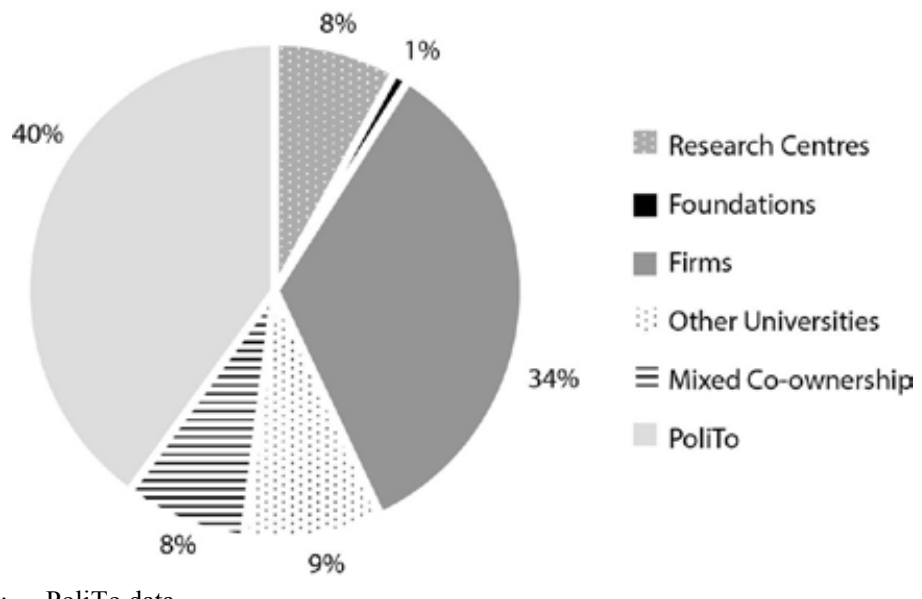

Source: PoliTo data.

Figure D.3 Patent co-ownership as a percentage of the total patent applications

The TRIN performs a range of activities across four broad categories:

(1) Research Commercialisation: With more than 600 registered patents and 53 university spin-off firms (in the last 15 years), PoliTo can be considered a leading university in technology transfer, both in Italy and the EU. The focus on collaboration with the ecosystem can be confirmed by the fact that around $50 \%$ of the patents are co-owned with local companies or research centres (Figure D.3) and there is a growing number of patent applications, but also patent licences to companies as well as the creation of spin-off firms (Figure D.4). In particular, there was an increase between 2015 and 2017, with 16 patents being licensed to firms and 23 to spin-offs. These technology transfer activities have generated 
resources for PoliTo of around $€ 1$ million from different sources and around $€ 0.7$ million of options on patents.

(2) Knowledge sharing: In order to increase the impact of research on the regional and national economy, PoliTo with the support of Banca Intesa and the Ministry of Economic Development, in 2016 set up a project aimed at creating a unique entry point (https://www.knowledge-share .eu/) for patents filed from most of the Italian universities and research centres. The objective of the Knowledge Share project is to increase the opportunities for SMEs to access the pool of patents (and the related technologies) in order to sustain their innovation processes.

(3) Support to technology development and spin-off creation: The general strategy of PoliTo as far as tech transfer is concerned is to provide support for 'inside-out' technology development and spin-off inception. The support provided by PoliTo, through TTO, consists of both 'soft' and 'hard' assistance: mentoring programmes, PoC funding, networking with local entrepreneurs, potential investors, or large companies. The incubator supports the growth of start-ups ${ }^{15}$ and further funding stages. PoliTo's ability to internally increase the Technology Readiness Level (TRL) of research results has attracted the attention and investments of business angels (they have co-invested in some PoCs), venture capitalists focused on tech transfer and early stages, as well as local medium-sized companies that are looking for new technological opportunities. One successful case is the ToothPic spin-off, which resulted from an ERC research project, and which was the first investment ever in Italy based on EIF (European Investment Funds), specifically targeted at university tech transfer.

(4) Collaborative research with industry and engagement with the ecosystem: As mentioned in section D.1, PoliTo invested $€ 30$ million in 2016 to create 11 cross-departmental research centres that perform research on 'breakthrough technologies' (e.g. applied photonics, additive manufacturing, water technologies, artificial intelligence and big data). These centres are at the intersection of different scientific disciplines and therefore need new approaches. Such research centres share their infrastructures with large companies and SMEs. In addition, in 2015, PoliTo initiated an array of programmes aimed at developing new forms of collaboration with SMEs in collaboration with local industrial associations and other local actors. These programmes provide scientific tutoring and coaching services to obtain product and/or process innovations in

15 It should be noted that the incubator is open to business ideas coming from outside the regional ecosystem as well. 
SMEs. On an annual basis, the number of involved companies in such programmes is more than 100 . Their objective has been to make PoliTo technologies available to a large number of SMEs. All these above activities have in fact been successful in turning knowledge from education and research into technology transfer (Table D.2).

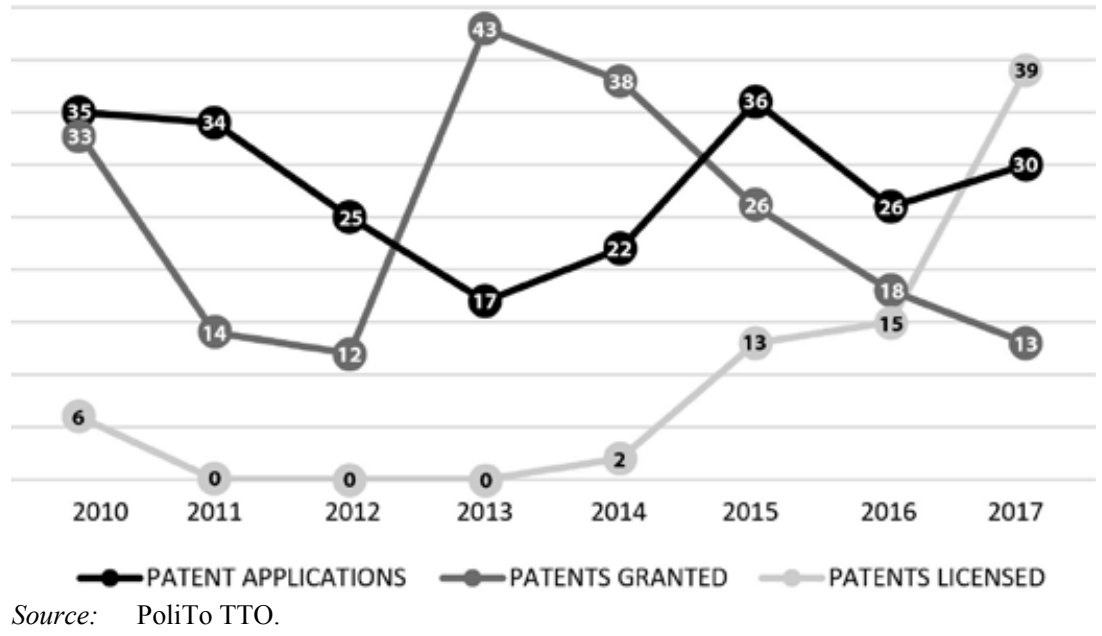

Figure D.4 Trends in patenting activities

Table D.2 Technology performance indicators

\begin{tabular}{lr}
\hline Invention disclosures since 2010 & 303 \\
Patent applications since 2010 & 241 \\
Patents granted since 2010 & 203 \\
Commercialised patents since 2010 & 80 \\
Patent co-ownership rate since 2010 & $59 \%$ \\
Spin-offs since 2004 & 54 \\
Total start-ups launched by the incubator & 224 \\
Total amount of funding raised by portfolio companies since 2010 & $€ 5 \mathrm{M}$ \\
\hline
\end{tabular}




\section{D.5 ENTERPRISE DEVELOPMENT AND ENTREPRENEURSHIP}

Enterprise development and entrepreneurship have been a key concern since 1999, the year in which I3P, ${ }^{16}$ a PoliTo incubator, was founded. This incubator is a non-profit joint-stock consortium that includes the Turin Chamber of Commerce, the City of Turin and the Province of Turin as shareholders. Since it was first set up, I3P has promoted and supported the creation and development of new enterprises by both PoliTo researchers, to exploit their scientific results, and by external entrepreneurs and/or established firms with new business ideas. The number of PoliTo spin-offs has increased gradually from 2004 to 2018 (Figure D.5), with a peak in 2009.

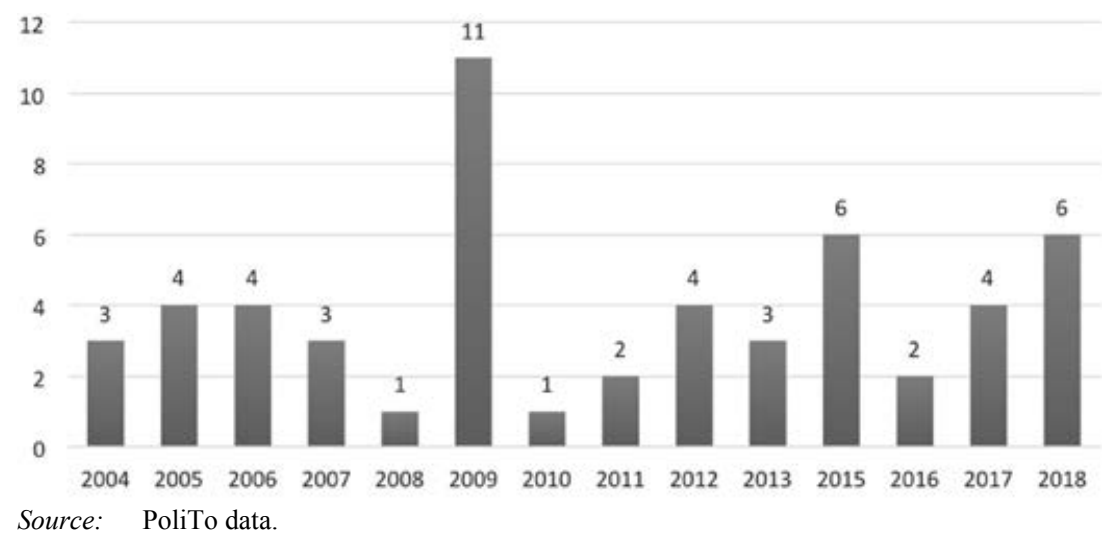

Figure D.5 Spin-off firms produced by PoliTo

I3P has in fact been successful in introducing new companies (Figure D.6). The number of incubated companies has increased substantially throughout the period from 2011 to 2018 . The incubated start-ups have determined a positive impact on the local economy and on employment. In fact, the total turnover and employment have risen gradually from 2011 to 2018, and, in 2016, they stood at 1687 and $€ 124$ million, respectively (Table D.3). Investment in start-ups has also grown in the same period, reaching a 2017 peak of $€ 3.02$ million and $€ 6.16$ million for seed and early stage investment respectively.

16 Incubatore Imprese Innovative del Politecnico di Torino (PoliTo Incubator for innovative firms). 
I3P has therefore played a very important role in accelerating the diffusion of innovations that were generated in the PoliTo labs as well as of business ideas originating from the entrepreneurial ecosystem. It works from TRL 5-6 on, and together with PoliTo has created an entrepreneurial climate inside the university and the region, by means of specific events and meetings with industrial representatives, investors and other members of the business community.

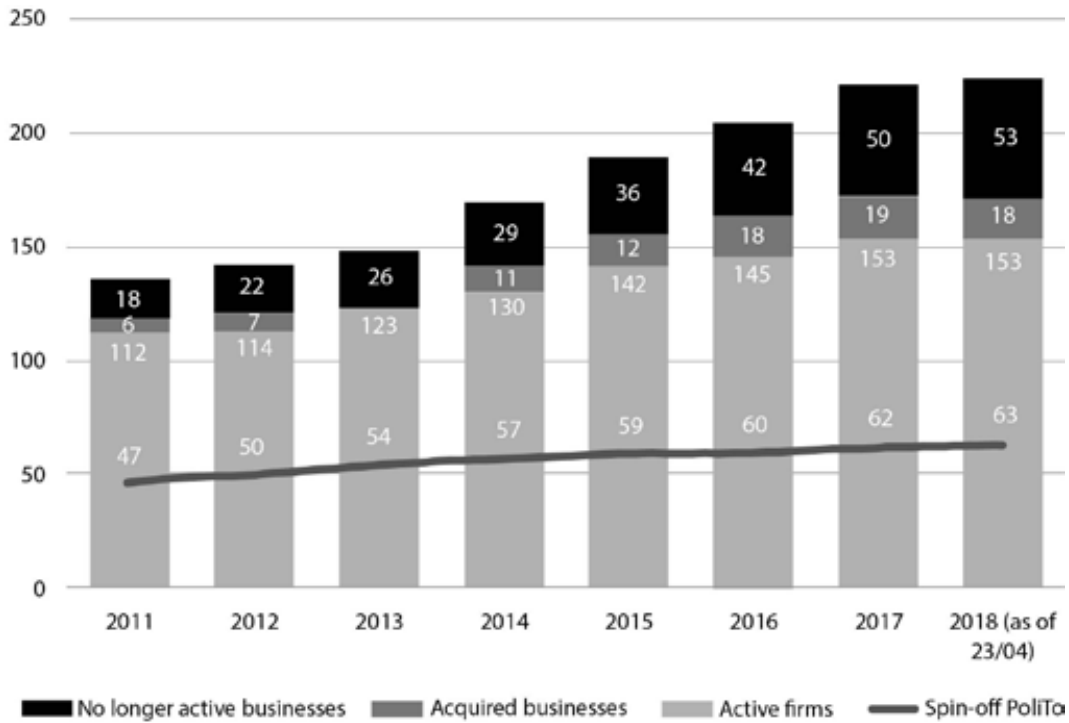

Figure D.6 Trend of start-ups incubated by I3P

Table D.3 I3P performance statistics

\begin{tabular}{lrrrrrrr}
\hline & 2011 & 2012 & 2013 & 2014 & 2015 & 2016 & 2017 \\
\hline Total employment created by & 672 & 778 & 1176 & 1408 & 1515 & 1687 & $2207^{1}$ \\
start-ups & & & & & & & \\
Patents owned by start-ups & 78 & 74 & 78 & 70 & 86 & 97 & $103^{1}$ \\
Total Turnover [M€] & 44 & 50 & 61 & 75 & 94 & 124 & 2 \\
Seed Investment [M€] & 1.7 & 1 & 2.3 & 3 & 3.2 & 2.9 & 3.02 \\
Early stage investment [M€] & 5 & - & - & 2.5 & 8.2 & 5.15 & 6.16 \\
\hline
\end{tabular}

Notes: $\quad{ }^{1}$ Estimated, ${ }^{2}$ Not available. 


\section{D.6 VISION AND STRATEGY FOR THE NEAR FUTURE}

In the future, PoliTo will continue its investments aimed at increasing the technological proximity with firms from the regional ecosystem. On one hand, the process is pulled by local firms with high innovation capabilities, and on the other pushed by research activities at PoliTo. In line with regional policies to achieve a balanced economy, it will continue its evolution from a strategy of exploiting competencies that are already part of the local industrial ecosystem, towards a more exploratory strategy aimed at supporting the emergence of new industries. Moreover, PoliTo will undertake the following steps:

- Expand the involvement of SMEs: Although some steps have already been undertaken, only a few SMEs are currently collaborating with the university. Since 2014, a more integrated approach has been adopted, and a growing number of initiatives have been implemented. The involvement of such SMEs will continue and expand in the near future, possibly together with the upcoming initiative concerning the Competence Centre (see the last point).

- Formalising technology transfer activities: The structure of TTO is being redesigned in order to strengthen the formal relationships between the PoliTo departments and external actors, as well as a revision of the procedures for IP protection and diffusion. This will favour the transfer of knowledge and technology, especially to SMEs, and will help support them in appropriating returns from collaborative R\&D.

- Expanding cross-departmental research centres in collaboration with industry - the creation of cross-department research centres, focused on new 'breakthrough technologies', will be completed in 2018. One of the key challenges will be how to share research infrastructures with both large companies and SMEs in order to allow them to co-evolve in the development of emerging technologies. TTO will be involved in this process, so that this collaborative research can be achieved.

- Increasing the attraction of investments: Activities related to the 'Third Mission' still need further investments in order to maintain the steady growth in the number of filed patents regarding new technologies, the growth in the number of spin-offs and their ability to raise early stage funding from investors and local companies. In this respect, the agreements signed in 2017-2018 with V3, ${ }^{17}$ a Venture Capitalist fund that specifically

17 Vertis Venture 3 Technology Transfer. 
targets university TT, represents a first remarkable attempt to attract investments in order to foster technology development and transfer.

- Creation of a new research collaborative space: In June 2018, PoliTo won a competitive national tender, financed by the Italian Minister of Economic Development, for the creation of a 'Competence Centre' focused on key enabling technologies regarding 'Industry 4.0'. Such technologies include the Internet of Things, Big Data, Blockchain, Additive Manufacturing and so on, and their development is aimed at creating a collaborative space with large firms and SMEs, where training and research collaboration in TRLs from 5 to 7 can be performed. 